\title{
Measurement Point Selection and Modal Damping Identification for Bladed Disks
}

\author{
Darren E. Holland* Jason R. Rodgers ${ }^{\dagger}$ \\ Bogdan I. Epureanu ${ }^{\ddagger}$ \\ University of Michigan, Ann Arbor, Michigan 48109 USA
}

\begin{abstract}
A novel measurement point selection (MPS) technique for bladed disks (blisks) is presented and applied to a new modal damping identification method. When gathering data to be used for applications such as mistuning identification in blisks, it is important to measure points which provide sufficient and accurate information for the analysis. However, to reduce the experimental time and cost, the measurement points should be chosen optimally so that the minimum number of measurements have to be collected. This paper discusses a modified form of the effective independence distribution vector (EIDV) method presented by Penny et al. and adapted by Holland et al. The key novel aspect of the proposed method is that it uses only single sector-level calculations instead of the whole system. A residual weighting optimizes the MPS technique for noisy measurements. The method presented is equivalent to the full system EIDV method, but it decreases the computational cost, increases the robustness of the identification, and minimizes the measurement time. Also, a novel method to identify damping parameters for each mode in a frequency range of interest is presented. This method utilizes the proposed MPS technique to increase the accuracy of the identification. Measurement locations and modal damping results for a 30 degree of freedom system and a blisk with a complex geometry are presented. Using the proposed methodologies it is possible to obtain an accurate modal damping identification with a decreased computational and measurement cost.
\end{abstract}

\section{Introduction}

Realistic structures such as bladed disks (blisks) have a complex geometry. Capturing the motion of such complex structures can be very difficult and typically involves finite element models with a large number of degrees of freedom (DOFs). These models employ parameters which are often not well known. One such parameter is damping. Thus, identification techniques are needed to determine the actual damping. These identification techniques require measurements of the vibratory response of the structure. In general, the more measurement points are used the more accurate the identification is. However, as the number of measurement locations increases, the cost of the experimental work increases greatly. Conceptually, the effective independence distribution vector (EIDV) method $^{1}$ determines the amount of modal information for each point in a candidate set of measurement locations. Holland et al. ${ }^{2}$ extended the EIDV method to determine the minimum number and the best locations of the necessary measurements. Those approaches used whole system models which required a heavy computational effort. In this work, an optimized measurement point selection (MPS) technique is introduced to reduce the computational effort for blisks and to increase the robustness of the selection process.

Small sector-to-sector variations (mistuning) in the mass or stiffness of blisks can cause an increase in the maximum vibration amplitudes and stresses compared to their analog cyclically symmetric (or tuned) structures. This increase can lead to high cycle fatigue failure. In an effort to minimize the effects of vibrations, damping coatings can be applied to these structures. As mistuning may significantly affect certain modes, the damping coatings should decrease the response of these modes. To determine the effectiveness of

*Graduate Student, Department of Mechanical Engineering, deholla@umich.edu

†Undergraduate Student, Department of Mechanical Engineering, rodgersr@umich.edu

¥Associate Professor, Department of Mechanical Engineering, epureanu@umich.edu 


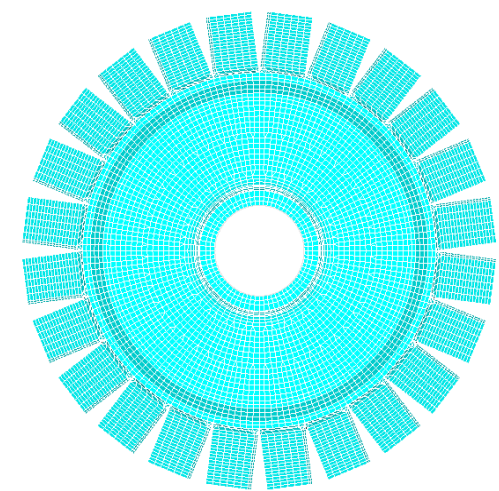

Figure 1. Cyclically symmetric model (blisk)

damping coatings, the modal damping of systems with and without the coating have to be determined. There are many methods for damping identification. ${ }^{3-16}$ In general, these techniques only apply to low dimensional systems or to systems with low modal density. These methods can be grouped into two categories. The methods in the first category require measuring damped eigenvalues and mode shapes, and do not require measurements of the excitation. The methods in the second category require constructing (full) frequency response functions, and therefore require knowledge of the excitation. Herein, a novel modal damping identification method is presented which accurately identifies the modal damping, is less sensitive to noise than other methods, works well with both full order and reduced order models (ROMs) for (low and) high modal density cases, and only requires knowledge of the relative forcing (i.e. forcing has be known only up to a multiplicative constant). Such forcing is obtained in many cases where the applied forcing is only known to be the same as a reference forcing, whereas the actual magnitude of the reference forcing is unknown. An example of such a case is the forcing calibrated by using the procedure proposed by Holland et al. ${ }^{2}$

Griffin et al. ${ }^{17}$ found that slightly mistuned system modes can be well represented as a linear combination of tuned system modes. This relationship has been used in studying the effects of crack propagation, ${ }^{18-20}$ and multi-stage phenomena. ${ }^{21}$ Therefore, the optimal measurement locations for a set of tuned system modes of a blisk (in a frequency range of interest) are also the best locations for the slightly mistuned blisk. Moreover, the damping identification does not require cyclic symmetry, but it assumes that the mass and stiffness matrices are already identified. ${ }^{22,23}$ Therefore, the optimal MPS and damping methods apply to both cyclically symmetric and slightly mistuned systems.

\section{MPS Extension}

In this section, the EIDV method is extended in three different ways. First, the EIDV procedure is modified to take advantage of cyclic symmetry. Next, a residual weighting which considers the effects of noise is added to the EIDV method. Third, an algorithm is introduced to determine the measurement locations when the number of candidate DOF is very large.

\section{A. EIDV and Cyclic Symmetry}

The EIDV-based procedure presented by Holland et al. ${ }^{2}$ uses the full tuned system modes to calculate the optimal measurement locations. When the model of interest has many sectors with many candidate measurement locations, the matrices involved can become prohibitively large. However, using cyclic symmetry and exploiting the properties of the matrices involved in the EIDV procedure, one may redefine the minimum EIDV value and transform the method as to require only single sector calculations. As a result, the matrix sizes reduce from the total number of DOFs in all sectors in the system to the number of DOFs in a single sector. Therefore, the computational and memory costs are dramatically reduced. Next, we discuss such an EIDV-based method with cyclic symmetry.

The EIDV matrix is defined as

$$
\mathbf{E I D V}_{a}^{\text {full }}=\operatorname{diag}\left(\boldsymbol{\Phi}\left[\boldsymbol{\Phi}^{T} \boldsymbol{\Phi}\right]^{-1} \boldsymbol{\Phi}\right)
$$


where $\boldsymbol{\Phi}$ is a subset of tuned system modes in a frequency range of interest. Many such subsets can be chosen. To refer to one of these subsets, the subscript $a$ is used to indicate the $a^{\text {th }}$ subset.

Note that $\mathbf{E I D V}_{a}^{\text {full }}$ is idempotent and symmetric, and

$$
\boldsymbol{\Phi}=\left(\mathbf{F} \otimes \mathbf{I}_{d \times d}\right) \boldsymbol{\Phi}_{s e c},
$$

where $\mathbf{F}$ is the real Fourier matrix of size $s \times s$ (with $s$ being the number of sectors in the system), $d$ is the number of candidate DOFs in one sector, and $\boldsymbol{\Phi}_{s e c}$ is a block diagonal matrix containing the $a^{\text {th }}$ subset of tuned system modes for a single sector. Each block in $\boldsymbol{\Phi}_{\text {sec }}$ corresponds to a different nodal diameter. Substituting Eq. 2 into Eq. 1, one obtains

$$
\mathbf{E I D V}_{a}^{\text {full }}=\operatorname{diag}\left(\left[\mathbf{F} \otimes \mathbf{I}_{d \times d}\right] \boldsymbol{\Phi}_{\text {sec }}\left[\boldsymbol{\Phi}_{\text {sec }}^{T} \boldsymbol{\Phi}_{s e c}\right]^{-1} \boldsymbol{\Phi}_{\text {sec }}^{T}\left[\mathbf{F} \otimes \mathbf{I}_{d \times d}\right]\right)
$$

where $\boldsymbol{\Phi}_{\text {sec }}\left[\boldsymbol{\Phi}_{\text {sec }}^{T} \boldsymbol{\Phi}_{\text {sec }}\right]^{-1} \boldsymbol{\Phi}_{\text {sec }}^{T}$ is idempotent. The trace of any idempotent matrix is equal to its rank. This key observation ${ }^{1}$ can be used to select as measurement DOFs the DOFs which correspond to the largest values on the diagonal of $\mathbf{E I D V}_{a}^{\text {full }}$. For a cyclically symmetric system, the entry of $\mathbf{E I D V}_{a}^{\text {full }}$ for a DOF on one sector is the same for all sectors. Hence, the same DOF that is chosen for one sector is also chosen as a measurement DOF for the all other sectors. Also, the rank of two matrices related through a similarity transformation is the same. Therefore, the cyclic expansion in Eq. 2 is not needed, and the first sector can provide all the required information. Now define

$$
\mathbf{E I D V}_{a}^{s e c}=\operatorname{diag}\left(\boldsymbol{\Phi}_{s e c}\left[\boldsymbol{\Phi}_{s e c}^{T} \boldsymbol{\Phi}_{s e c}\right]^{-1} \boldsymbol{\Phi}_{s e c}^{T}\right) .
$$

Since $\boldsymbol{\Phi}_{s e c}$ is block diagonal,

$$
\mathbf{E I D V}_{a}^{s e c}=\operatorname{diag}\left[\begin{array}{ccc}
\ddots & \mathbf{0} & \mathbf{0} \\
\mathbf{0} & \mathbf{A}_{z}\left[\mathbf{A}_{z}^{T} \mathbf{A}_{z}\right]^{-1} \mathbf{A}_{z}^{T} & \mathbf{0} \\
\mathbf{0} & \mathbf{0} & \ddots
\end{array}\right]
$$

where $\mathbf{A}_{z}$ is the $z^{\text {th }}$ block of the $a^{\text {th }}$ subset of tuned sector modes corresponding to the $z^{\text {th }}$ nodal diameter. Each block of $\mathbf{E I D V}_{a}^{\text {sec }}$ is an idempotent matrix, where the diagonal values are the contribution of each DOF to the linear independence of that block of modes. Let

$$
\mathbf{d}_{a, z}=\operatorname{diag}\left(\mathbf{A}_{z}\left[\mathbf{A}_{z}^{T} \mathbf{A}_{z}\right]^{-1} \mathbf{A}_{z}^{T}\right),
$$

where diag refers to the diagonal entries of the enclosed matrix. The values in the vector $\mathbf{d}_{a, z}$ are the candidate DOF in one sector for the modes from $\boldsymbol{\Phi}_{\text {sec }}$ which have nodal diameter $z$. These values need to be combined to determine the overall EIDV value. For nodal diameters with mode pairs, $\mathbf{d}_{a, z}$ contains two values for each candidate DOF (otherwise $\mathbf{d}_{a, z}$ only contains one value per DOF). Therefore, let $\overline{\mathbf{d}}_{a, z}$ be a matrix where the $b^{\text {th }}$ column contains the $b^{\text {th }}$ occurrence of the candidate DOF in $\mathbf{d}_{a, z}$. If nodal diameter $z$ has a single mode (not a pair), then $\overline{\mathbf{d}}_{a, z}=\mathbf{d}_{a, z}$. Define the new EIDV value to be

$$
\operatorname{EIDV}_{a}=\sum_{z} \sum_{b} \overline{\mathbf{d}}_{a, z}^{b}
$$

where $b$ is 2 for nodal diameters with mode pairs, and 1 otherwise. The DOF corresponding to the minimum value of $\mathbf{E I D V}_{a}$ contributes the least to the linear independence of the nodal diameters. Therefore, the sum of these values is an indicator of the contribution to the linear independence of the candidate DOFs for the modes in the frequency range of interest. Similar to the original EIDV method, ${ }^{1}$ the MPS approach eliminates as a candidate DOF the DOF (one on each sector) which corresponds to the lowest value in $\operatorname{EIDV}_{a}$.

\section{B. Residual Weighting}

To be robust and to provide accurate results, a MPS method must be insensitive to measurement noise. Therefore, we introduce a residual weighting which examines the error (associated with each measurement 
location) caused by noisy measurements. The MPS (with residual weighting) is normally used for both mistuning identification (not discussed here) as well as damping identification. First, a numerical forced response is performed at frequencies $\omega_{y}$ near the system natural frequencies. For increased computational efficiency, a ROM is used. To ensure that the selected measurement points are statistically optimal for any mistuning pattern (with some known maximum mistuning in each blade), multiple forced responses are calculated. Each forced response is calculated for a random level of mistuning and cyclic modeling error ${ }^{22}$ within model specific limits (e.g. between $-5 \%$ and $+5 \%$ mistuning). Noise is then injected into the calculated response to obtain surrogate data which mimic experimental data. Let $X_{\text {noisy }}^{q}\left(\omega_{y}\right)$ be the forced response of DOF $q$ after noise has been injected into the actual (exact) response $X_{a c t}^{q}\left(\omega_{y}\right)$. Then, low responding data is removed from both the actual and the noisy data. Next, for each $\omega_{y}$, the noisy responses of all candidate DOF $q$ are grouped in a vector $\mathbf{X}_{n o i s y}\left(\omega_{y}\right)$. Let $\mathbf{X}_{\text {trans }}\left(\omega_{y}\right)=\boldsymbol{\Phi}\left[\boldsymbol{\Phi}^{T} \boldsymbol{\Phi}\right]^{-1} \boldsymbol{\Phi}^{T} \mathbf{X}_{n o i s y}\left(\omega_{y}\right)$. The residual weighting for the $q^{\text {th }}$ candidate measurement DOF is defined as

$$
\operatorname{Res}_{a}^{q}=\frac{\epsilon+\min _{q=1 \ldots D O F}\left(\sum_{y}\left|X_{a c t}^{q}\left(\omega_{y}\right)-X_{\text {trans }}^{q}\left(\omega_{y}\right)\right|\right)}{\epsilon+\sum_{y}\left|X_{a c t}^{q}\left(\omega_{y}\right)-X_{\text {trans }}^{q}\left(\omega_{y}\right)\right|},
$$

where $|\cdot|$ denotes the absolute value. The residual in Eq. 5 contains information about the sensitivity of the measurement locations to noise (when a specific ROM is used). Note that $\operatorname{Res}_{a}^{q}$ is a positive value between zero and 1. A low value of $\operatorname{Res}_{a}^{q}$ (close to zero) corresponds to a high sensitivity to noise. A high value of $\operatorname{Res}_{a}^{q}$ (close to 1) corresponds to a low sensitivity to noise.

For DOFs with low values in the residual weighting, the MPS becomes dominated by the residual weighting. For DOFs with high values in the residual weighting, the MPS is dominated by the EIDV method. To achieve a balance between the noise sensitivity of each DOF and their contribution to the linear independence of the measurements, $\epsilon$ is defined as

$$
\epsilon=\operatorname{mean}_{q=1 \ldots D O F, y}\left|X_{a c t}^{q}\left(\omega_{y}\right)\right| .
$$

In Eq. 5, the residual of the transformation from the physical to modal and back to physical coordinates $\left|X_{a c t}^{q}\left(\omega_{y}\right)-X_{\text {trans }}^{q}\left(\omega_{y}\right)\right|$ is calculated, and the mean value over the surrogate and noise realizations is retained. As each sector contains the same DOF, the minimum residual weighting over each blade becomes the final residual weighting vector. This weighting cannot be done using sector-level calculations. However, as the residual is only calculated one time (for the set of all candidate DOF) the residual weighting is less expensive computationally than the EIDV method.

The new MPS method combines the EIDV method with the residual weighting. Let

$$
M P S_{a}^{q}=\operatorname{Res}_{a}^{q} E I D V_{a}^{q} .
$$

The minimum $M P S_{a}^{q}$ corresponds to the DOF $q$ (one on each blade) which contributes the least to the linear independence and is more sensitive to noise than the other candidate locations. The best measurement locations for different ROMs can be easily obtained by determining $M P S_{a}^{q}$ for $q=1 \ldots D O F$ for the corresponding set $a$ of modes in each ROM.

\section{Optimized MPS}

Next, the process of determining the best set of measurement points is described using an optimized MPS technique, as shown in Fig. 2. This algorithm is particularly important when there is a large number of candidate DOF because reducing the memory usage and the calculation cost are often necessary in such cases.

First, a set of candidate measurement locations is chosen. These locations should contain regions on the structure which are considered to be of interest and are physically possible to measure. Such locations may be chosen from a finite element model with a very fine mesh. Thus, it may be necessary to further sub-select from the candidate set. To do this sub-selection, the geometric information of the candidate points is used to choose a smaller set of locations where the reduced candidate measurement locations are uniformly distributed over the structure. From the reduced candidate measurement locations, the MPS method determines an initial 


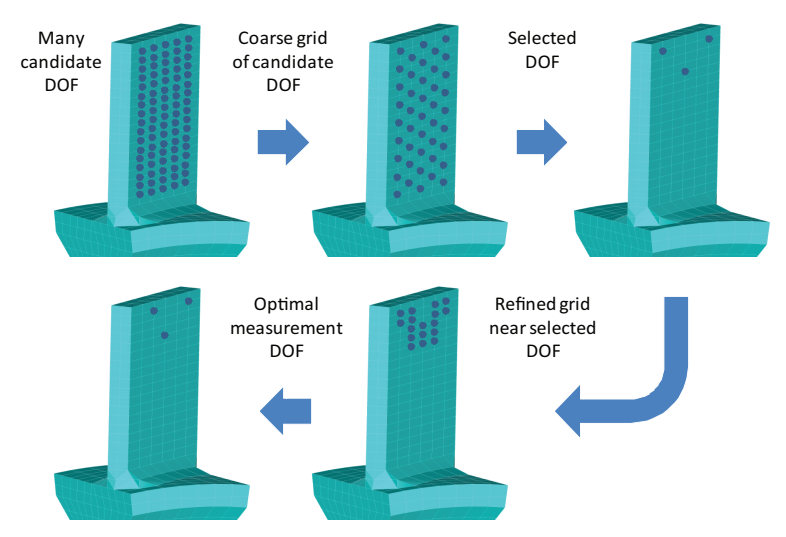

Figure 2. Optimal MPS algorithm

set of measurement locations. If desired, a local refinement step can be performed as follows. Taking all candidate locations (from the original set) that are in a region close to the previously chosen measurement points, one can define a new reduced set of candidate measurement locations. Applying the MPS method again on this new candidate set (containing only points near the previously chosen measurement points) provides a refined, optimal set of measurement locations.

\section{Modal Damping}

The measurement points chosen using the algorithm in the previous section can be used for structural identification. In particular, a novel modal damping identification method is presented next. This method identifies a damping parameter for each mode in a ROM. The formulation applies to both tuned and mistuned systems.

\section{A. Modal Damping Model}

The equations of motion for a linear structure can be expressed as

$$
\mathbf{M} \ddot{\mathbf{x}}+\mathbf{C} \dot{\mathbf{x}}+\mathbf{K x}=\overline{\mathbf{f}}
$$

where $\mathbf{M}$ is the mass matrix, $\mathbf{C}$ is the damping matrix, $\mathbf{K}$ is the stiffness matrix, $\mathbf{x}$ is the response, and $\overline{\mathbf{f}}$ is the applied forcing. This forcing is assumed harmonic with amplitude $\mathbf{f}$ and frequency $\omega$. Therefore, the physical response $\mathbf{x}$ is harmonic and has a magnitude $\mathbf{X}$ given by

$$
-\omega^{2} \mathbf{M X}+j \omega \mathbf{C X}+\mathbf{K X}=\mathbf{f}
$$

where $j$ is the imaginary unit.

Next, $\mathbf{X}$ is converted to tuned (or mistuned) modal coordinates $\mathbf{X}=\boldsymbol{\phi} \mathbf{p}$, where $\boldsymbol{\phi}$ is a matrix containing the undamped tuned (or mistuned) system normal modes. The matrix $\phi$ is of size $N \times N$, where $N$ is the total number of DOF in the model (and is also the total number system modes). Note that for a mistuned system, $\phi$ can be computed also as the transformation from physical coordinates to mistuned coordinates under the assumption that the mistuned modes are a linear combination of tuned system modes. ${ }^{24}$ Pre-multiplying Eq. 7 by $\phi^{T}$, one obtains

$$
-\omega^{2} \mathbf{p}+j \omega \boldsymbol{\phi}^{T} \mathbf{C} \boldsymbol{\phi} \mathbf{p}+\boldsymbol{\Lambda} \mathbf{p}=\boldsymbol{\phi}^{T} \mathbf{f},
$$

where the system modes are mass normalized, and $\boldsymbol{\Lambda}=\boldsymbol{\phi}^{T} \mathbf{K} \boldsymbol{\phi}$ is a diagonal matrix containing the tuned (or mistuned) eigenvalues.

Let $\mathbf{C}=2 \mathbf{M} \boldsymbol{\phi} \operatorname{diag}\left(\zeta_{u} / \omega_{n}^{u}\right) \boldsymbol{\phi}^{T} \mathbf{K}$, where $\zeta_{u}$ is the viscous modal damping coefficient for mode $u$, and $\omega_{n}^{u}$ is the undamped natural frequency of mode $u$. For simplicity, let $\zeta=\operatorname{diag}\left(\zeta_{u} \omega_{n}^{u}\right)$. Next, assume that outside a given frequency range of interest, $\mathbf{p}_{v} \approx 0$ for a known index set $v$. Then the $v$ columns of $\boldsymbol{\phi}$ can be ignored. So, $\phi$ reduces to a matrix $\boldsymbol{\Phi}$ of size $N \times m$, where $m$ represents the number of modes in the frequency range 
of interest. These $m$ modes are used to construct ROMs in the frequency range of interest. The forced response can be obtained by solving

$$
-\omega^{2} \mathbf{p}+2 j \omega \zeta \mathbf{p}+\Lambda \mathbf{p}=\boldsymbol{\Phi}^{T} \mathbf{f}
$$

for $\mathbf{p}$. Next, converting from $\mathbf{p}$ to $\mathbf{X}$, one obtains

$$
\mathbf{X}=\boldsymbol{\Phi}\left[-\omega^{2} \mathbf{I}+2 j \omega \boldsymbol{\zeta}+\boldsymbol{\Lambda}\right]^{-1} \boldsymbol{\Phi}^{T} \mathbf{f}
$$

\section{B. Modal Damping Identification}

For excitation at frequencies $\omega_{i}$ and $\omega_{k}$ with forcing such that $\mathbf{f}_{i}=\alpha_{k} \mathbf{f}_{k}$ (with known $\alpha_{k} \in \mathbb{C}$ ), Eq. 9 becomes

$$
-\omega_{i}^{2} \mathbf{p}_{i}+2 j \omega_{i} \zeta \mathbf{p}_{i}+\boldsymbol{\Lambda} \mathbf{p}_{i}=\boldsymbol{\Phi}^{T} \mathbf{f}_{i}, \quad \text { and } \quad-\omega_{k}^{2} \alpha_{k} \mathbf{p}_{k}+2 j \omega_{k} \boldsymbol{\zeta} \alpha_{k} \mathbf{p}_{k}+\boldsymbol{\Lambda} \alpha_{k} \mathbf{p}_{k}=\boldsymbol{\Phi}^{T} \alpha_{k} \mathbf{f}_{k} .
$$

Such forcing is obtained in many cases where the applied forcing is only known to be the same as a reference forcing, whereas the actual magnitude of the reference forcing is unknown. An example of such a case is the forcing calibrated by using the procedure proposed by Holland et al. ${ }^{2}$ In that case $\alpha_{k}=1$. Rearranging, Eq. 11 becomes

$$
2 j \boldsymbol{\zeta}\left(\omega_{i} \mathbf{p}_{i}-\alpha_{k} \omega_{k} \mathbf{p}_{k}\right)=\omega_{i}^{2} \mathbf{p}_{i}-\omega_{k}^{2} \alpha_{k} \mathbf{p}_{k}-\boldsymbol{\Lambda}\left(\mathbf{p}_{i}-\alpha_{k} \mathbf{p}_{k}\right) .
$$

Rearranging $\boldsymbol{\zeta}\left(\omega_{i} \mathbf{p}_{i}-\alpha_{k} \omega_{k} \mathbf{p}_{k}\right)$ and recalling the definition of $\boldsymbol{\zeta}$, one obtains

$$
2 j \operatorname{diag}\left(\omega_{n}^{u}\left[\omega_{i} p_{i u}-\alpha_{k} \omega_{k} p_{k u}\right]\right)\left[\begin{array}{c}
\zeta_{1} \\
\vdots \\
\zeta_{N}
\end{array}\right]=\omega_{i}^{2} \mathbf{p}_{i}-\omega_{k}^{2} \alpha_{k} \mathbf{p}_{k}-\boldsymbol{\Lambda}\left(\mathbf{p}_{i}-\alpha_{k} \mathbf{p}_{k}\right),
$$

where $\operatorname{diag}\left(\omega_{n}^{u}\left[\omega_{i} p_{i u}-\alpha_{k} \omega_{k} p_{k u}\right]\right)$ is a diagonal matrix with the $u^{\text {th }}$ diagonal entry given by $\omega_{n}^{u}\left[\omega_{i} p_{i u}-\alpha_{k} \omega_{k} p_{k u}\right]$. The viscous modal damping identification equation is then obtained as

$$
\left[\begin{array}{c}
\zeta_{1} \\
\vdots \\
\zeta_{N}
\end{array}\right]=\operatorname{Im}\left\{\operatorname{diag}\left(2 \omega_{n}^{u}\left[\omega_{i} p_{i u}-\alpha_{k} \omega_{k} p_{k u}\right]\right)^{-1}\left[\omega_{i}^{2} \mathbf{p}_{i}-\omega_{k}^{2} \alpha_{k} \mathbf{p}_{k}-\boldsymbol{\Lambda}\left(\mathbf{p}_{i}-\alpha_{k} \mathbf{p}_{k}\right)\right]\right\}
$$

and holds for all pairings of frequencies $\omega_{i}$ and $\omega_{k}$ as long as $\omega_{i} p_{i u}-\alpha_{k} \omega_{k} p_{k u} \neq 0$.

To eliminate zero-mean noise, the mean of Eq. 12 can be computed over all available frequency pairings measured. One obtains

$$
\left[\begin{array}{c}
\zeta_{1} \\
\vdots \\
\zeta_{N}
\end{array}\right]=\operatorname{Im}\left\{\operatorname{diag}\left(2 \omega_{n}^{u}\left\langle\omega_{i} p_{i u}-\alpha_{k} \omega_{k} p_{k u}\right\rangle\right)^{-1}\left[\left\langle\omega_{i}^{2} \mathbf{p}_{i}-\omega_{k}^{2} \alpha_{k} \mathbf{p}_{k}\right\rangle-\boldsymbol{\Lambda}\left\langle\mathbf{p}_{i}-\alpha_{k} \mathbf{p}_{k}\right\rangle\right]\right\}
$$

where $<\cdot>$ denotes the mean over all the frequency pairings.

\section{Identification Filtering}

To increase the accuracy of the modal damping identification, four identification filtering techniques are proposed. The first two filters eliminate measurement data that can cause erroneous solutions. The first of these filters removes low responding data, which can be greatly affected by noise. Responses with magnitudes below a fraction $T_{l}$ of the maximum response magnitude are removed. That is, measurements $X_{\text {meas }}^{q}\left(\omega_{y}\right)$ (collected at DOF $q$ for an excitation with frequency $\omega_{y}$ ) which satisfy

$$
\max _{q=1 \ldots D O F}\left|X_{\text {meas }}^{q}\left(\omega_{y}\right)\right|<T_{l} \max _{q=1 \ldots D O F, y}\left|X_{\text {meas }}^{q}\left(\omega_{y}\right)\right|
$$

are removed. The superscript $q$ represents the measurement DOFs.

The second filter removes measurements which have a relative error greater than a desired threshold $R_{\text {thresh }}$ in the transformation from physical to modal and back to physical coordinates. The measurements 


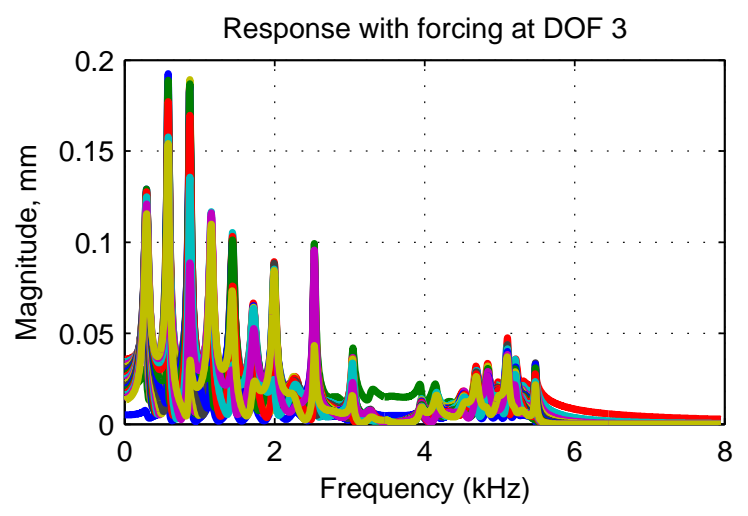

Figure 3. Sample forced response for the 30 DOF system

collected at frequencies $\omega_{y}$ for all $q=1 \ldots D O F$ are grouped in a vector $\mathbf{X}_{\text {meas }}\left(\omega_{y}\right)$. Measurements $\mathbf{X}_{\text {meas }}\left(\omega_{y}\right)$ which satisfy

$$
\begin{gathered}
\left\|\boldsymbol{\Phi}\left[\boldsymbol{\Phi}^{T} \boldsymbol{\Phi}\right]^{-1} \boldsymbol{\Phi}^{T} \operatorname{Re}\left(\mathbf{X}_{\text {meas }}\left(\omega_{y}\right)\right)-\operatorname{Re}\left(\mathbf{X}_{\text {meas }}\left(\omega_{y}\right)\right)\right\|_{2}>R_{\text {thresh }} \| \text { Re }\left(\mathbf{X}_{\text {meas }}\left(\omega_{y}\right)\right) \|_{2} \text { or } \\
\left\|\boldsymbol{\Phi}\left[\boldsymbol{\Phi}^{T} \boldsymbol{\Phi}\right]^{-1} \boldsymbol{\Phi}^{T} \operatorname{Im}\left(\mathbf{X}_{\text {meas }}\left(\omega_{y}\right)\right)-\operatorname{Im}\left(\mathbf{X}_{\text {meas }}\left(\omega_{y}\right)\right)\right\|_{2}>R_{\text {thresh }}\left\|\operatorname{Im}\left(\mathbf{X}_{\text {meas }}\left(\omega_{y}\right)\right)\right\|_{2}
\end{gathered}
$$

are removed, where Re and $I m$ denote real and imaginary parts, and $\|\cdot\|_{2}$ indicates the 2-norm.

The third and fourth filters remove from identification the damping for modes which are either not responding or have modal amplitudes which cannot be correctly determined. The third filter is based on the $i^{\text {th }}$ modal response resulting in negligible modal information. Responses which are too low (based on a maximum modal response threshold $\left.P_{\text {thresh }}\right)$ are considered to be too sensitive to noise. Thus, if mode $u$ has an amplitude $p_{i u}$ such that $\left|p_{i u}\right| \leq 0.5 \max _{r}\left|p_{i r}\right|$ (where $r=1 \ldots m$ ), or $\left|p_{i u}\right|<P_{\text {thresh }}$, then mode $u$ is ignored. The fourth and last filter removes measurements where the $i^{\text {th }}$ and $k^{\text {th }}$ modes are similar. Therefore, a pair of measurements $(i, k)$ is removed when

$$
\left|p_{i u}-\alpha_{k} p_{k u}\right| \leq P_{\text {thresh }}
$$

\section{Results}

In this section, the proposed method is validated using a 30 DOF system and the University of Michigan validation blisk. The 30 DOF spring-mass system has mass and stiffness matrices

$$
\mathbf{M}=\operatorname{diag}\left(\left[\begin{array}{llll}
1 & 2 & \cdots & 1
\end{array}\right]\right) \mathrm{kg},
$$

and

$$
\mathbf{K}=\left[\begin{array}{cccccc}
2.4 \cdot 10^{9} & -0.6 \cdot 10^{9} & & & & \mathbf{0} \\
-0.6 \cdot 10^{9} & 1.2 \cdot 10^{9} & -0.6 \cdot 10^{9} & & & \\
& & \ddots & & & \\
\mathbf{0} & & & -0.6 \cdot 10^{9} & 1.2 \cdot 10^{9} & -0.6 \cdot 10^{9} \\
& & & & -0.6 \cdot 10^{9} & 1.9 \cdot 10^{9}
\end{array}\right] \frac{\mathrm{kg}}{\mathrm{s}^{2}}
$$

where $\operatorname{diag}([\cdot])$ indicates a diagonal matrix with entries given by the included vector $[\cdot]$. Figure 3 shows a sample forced response where the excitation was applied to DOF 3 . Responses below $4 \mathrm{kHz}$ are in a region of low modal density, whereas responses at frequencies higher than $4 \mathrm{kHz}$ correspond to high modal density.

A ROM was created using modes 19 to 29 (ordered by frequency) with 20 DOF measured using the proposed MPS technique. These 11 modes were chosen since they are closely spaced, and therefore the damping is more challenging to identify. The values in the diagonal damping matrix $\mathbf{C}$ for this ROM are displayed in Fig. 4a. A force of magnitude $10 \mathrm{~N}$ and phase of 0 was applied to each measured DOF near 


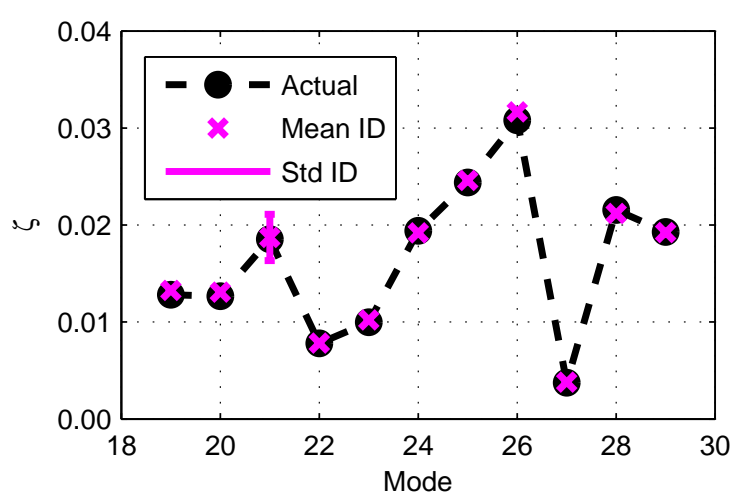

(a) Damping ID with $1 \%$ noise and optimal MPS

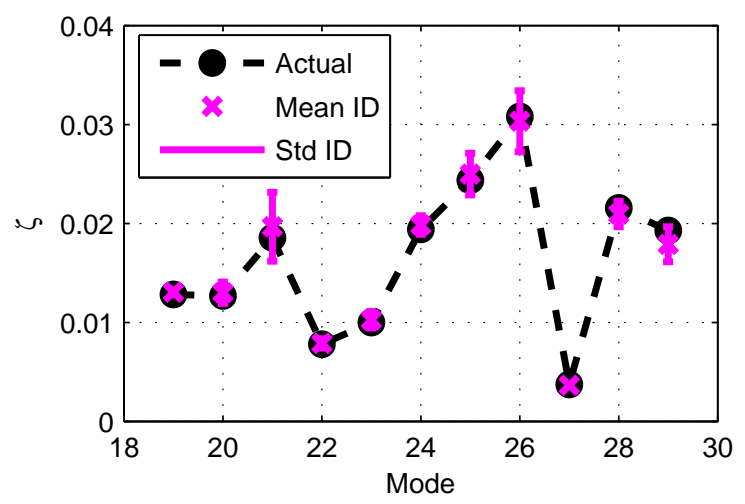

(c) Damping ID with $10 \%$ noise and non-optimal MPS

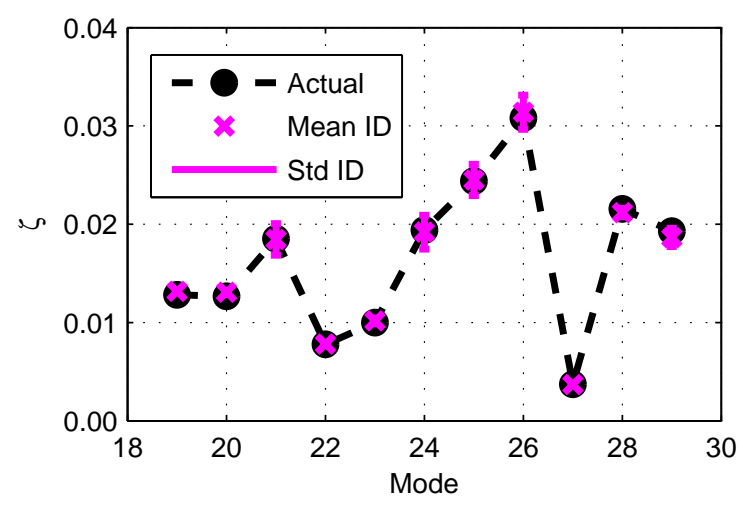

(b) Damping ID with $5 \%$ noise and optimal MPS

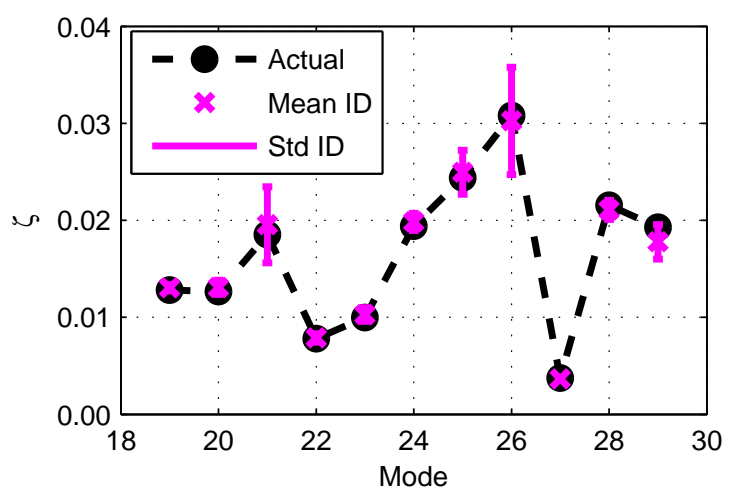

(d) Damping ID with $10 \%$ noise and optimal MPS

Figure 4. Damping ID for 30 DOF system

the natural frequency of each mode, and the physical response $X_{\text {meas }}^{q}\left(\omega_{y}\right)$ was recorded at frequencies $\omega_{y}$ for all measured DOF $q$. To simulate a physical experiment, measurement noise was injected using

$$
X_{\text {noisy }}^{q}\left(\omega_{y}\right)=X_{\text {meas }}^{q}\left(\omega_{y}\right)+N_{p} R_{1}^{q} X_{\text {meas }}^{q}\left(\omega_{y}\right)+\frac{\sqrt{2}}{2} N_{p} R_{2}^{q} \max _{q=1 \ldots D O F, y}\left(X_{\text {meas }}^{q}\left(\omega_{y}\right)\right)
$$

where $N_{p}$ is the maximum relative noise introduced, $R_{1}^{q}$ are randomly generated numbers drawn from a uniform distribution between -1 and 1 , and $R_{2}^{q}$ are complex numbers composed of real and imaginary parts with values drawn from a uniform distribution between 0 and $1 . R_{1}^{q}$ introduces relative measurement noise, whereas $R_{2}^{q}$ introduces absolute and bias noise.

The characteristics and validation of the measurement point selection and damping identification are presented for the 30 DOF system first. The following results show the mean and standard deviation of 1,000 identifications using different noisy measurement sets. The same 1,000 noise realizations $\left(R_{1}^{q}\right.$ and $R_{2}^{q}$ separately chosen for each $q$ DOF) were used to generate each of the following graphs. Standard deviation error bars are provided in the figures and are denoted by Std. Also, the filtering thresholds in the identification are $R_{\text {thresh }}=0.3, T_{l}=0.35$, and $P_{\text {thresh }}=1.0 \times 10^{-5}$.

The results in Fig. 4a-4d demonstrate that the 30 DOF damping identification is accurate using a ROM and for regions of high modal density. Figures $4 \mathrm{a}$ and $4 \mathrm{~b}$ show that the identified damping values are accurate when the noise level is low. The non-optimal and optimal MPS chose the same measurement locations for both $1 \%$ and $5 \%$ noise. Figures $4 \mathrm{c}$ and $4 \mathrm{~d}$ show that as the noise level increases, the optimal MPS chooses two different locations compared to the non-optimal MPS. The optimal MPS balances linear independence with sensitivity to noise. Therefore, the damping identified for the modes which are insensitive to noise may be less accurate than the damping identified using the non-optimal MPS, whereas the damping identified for modes which are more sensitive to noise should be more accurate. This change in accuracy can be seen in Figs. 4c and 4d, where the damping values for modes 20, 23, and 28 are more accurately identified with 


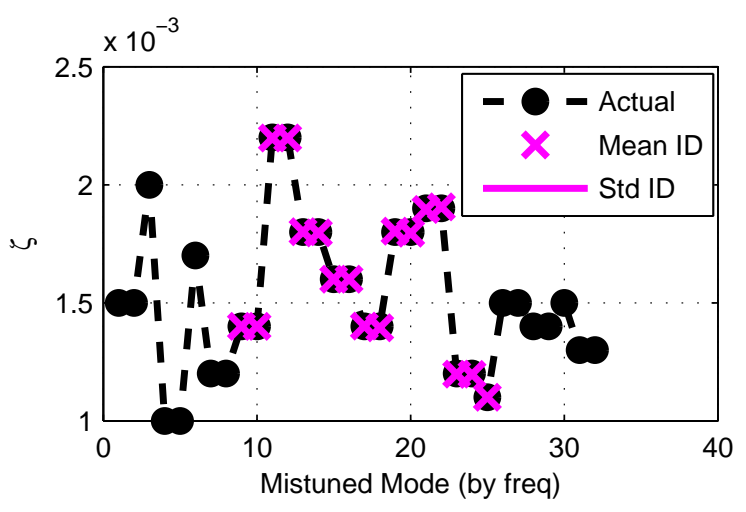

(a) Damping ID with $1 \%$ noise

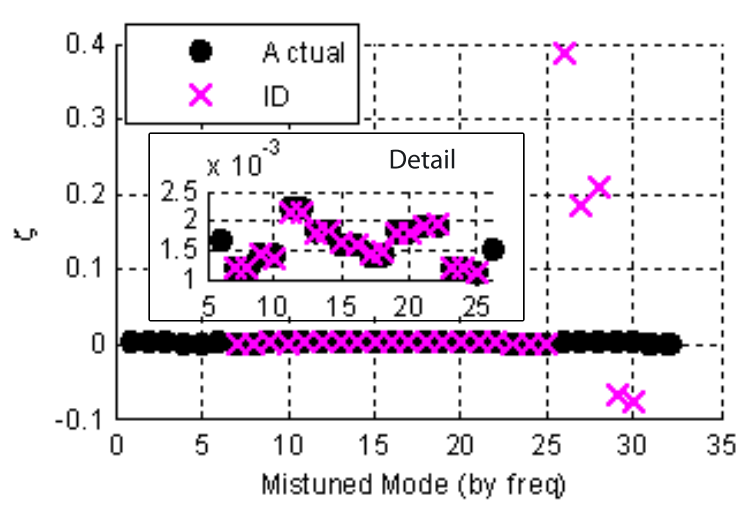

(c) Damping ID with $5 \%$ noise and 24 modes

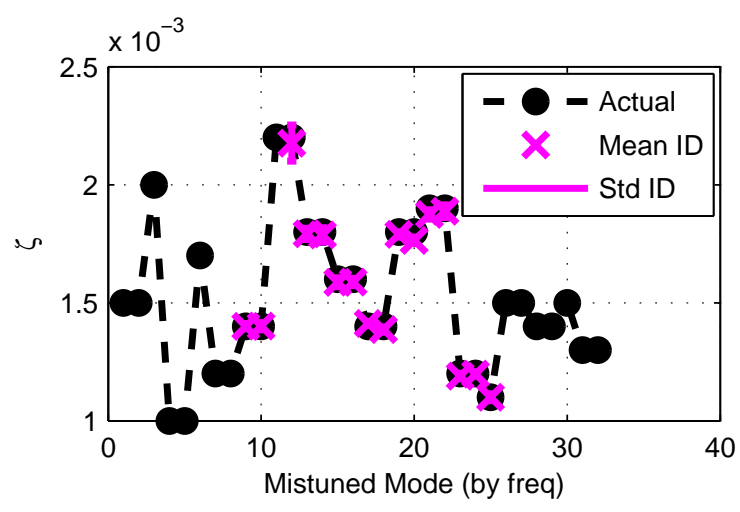

(b) Damping ID with $5 \%$ noise

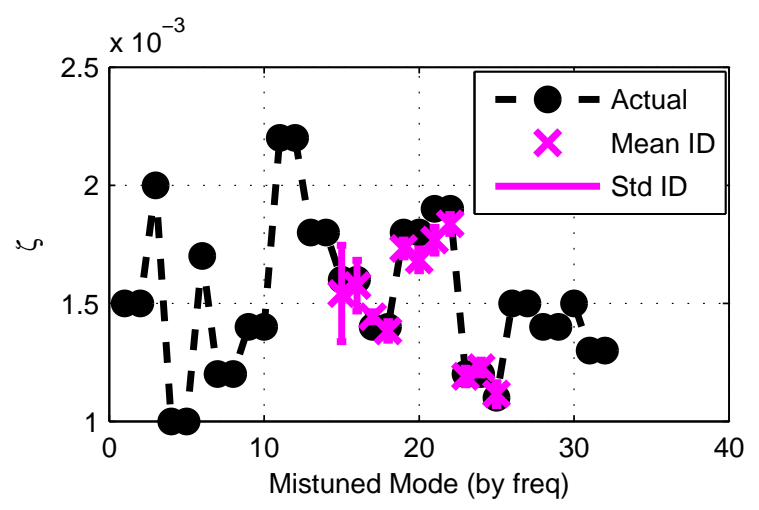

(d) Damping ID with $10 \%$ noise

Figure 5. Damping ID for validation blisk

the optimal MPS, but less accurately identified for modes 21, 25, and 26. Note that the standard deviation of most identified damping values is less than the level of the noise (e.g., the standard deviation is less than $10 \%$ for $10 \%$ noise in Figs. 4c and 4d). Modes 21 and 26 have a standard deviation greater than the level of the noise. Some of the mean damping values in Fig. 4a slightly exceed the 1\% noise level. However, all of the mean values have errors below the corresponding noise levels in Figs. 4b, 4c, and 4d. In general, the proposed method accurately identifies the modal damping with an error less than the corresponding noise level. Also, the optimal MPS improves the accuracy of the damping identification.

Care needs to be exercised when choosing the filtering parameters. If the filtering is too lax, the identification uses measurements that have less accurate modal coordinates due to a larger amount of noise in the response. Therefore, the identification becomes more contaminated by the noise effects. However, if the filtering is too strict, then the identification depends on too few measurements. As a result, the identification is also less accurate (since zero mean noise is not eliminated). The results presented are from the region of filtering which provides a balance between using measurements with less modal participation (of the modes for which the damping is to be identified) and elimination of zero mean noise. Note that different systems and measurements may require different values for $R_{\text {thresh }}, T_{l}$, and $P_{\text {thresh }}$.

Next, results for a more complex structure (in the form of the University of Michigan validation blisk ${ }^{25}$ ) are presented. This blisk has mistuning of approximately $2 \%$ (composed of $1 \%$ zero mean mistuning and $1 \%$ cyclic modeling error ${ }^{2}$ ). The damping identification uses the actual mistuning to assess the accuracy of the proposed measurement point selection and damping identification methods. The following results show the mean and standard deviation of 100 identifications using different noisy measurement sets.

The results in Fig. 5a-5d demonstrate that the mistuned validation blisk damping identification is accurate for regions of high modal density, uses a ROM, and typically has an error less than the measurement noise level. Figures $5 \mathrm{a}$ and $5 \mathrm{~b}$ display the identification results where $1 \%$ and $5 \%$ noise respectively have been injected into the numerically generated measurement data. For these figures, the ROM contained 17 modes. 
The damping was successfully obtained with a level of error in the identified damping similar to the level of the measurement noise. Both the standard deviation and mean damping values in Figs. 5a and 5b are well below the corresponding noise level. Figure 5d displays the identification results for $10 \%$ noise. The standard deviations and mean damping values for all modes except mode 15 are below the $10 \%$ noise level. Note that some modes in Figs. 5b and 5d were not identified. That is because the increase in the noise level made the identification filtering either remove all the measurement data or determine that the missing modes would not be accurately identified.

The effect of the size of the ROM used for identification can also be explored. Figure $5 \mathrm{c}$ shows that using a larger ROM causes errors in the identified damping of some modes (due to loss of rank in the $\boldsymbol{\Phi}$ matrix), although most modes (7 to 25) are accurately identified. Therefore, a ROM with greater size requires different measurement locations drawn from a larger candidate set. That is likely because modes in the larger ROM include disk motion. Hence, measurements which include the disk are needed for larger ROMs.

This behavior of the ROMs has already been explored in the context of mistuning identification. ${ }^{22}$ When introducing the concept of inverse ROMs (IROMs) for system identification, Madden et al. ${ }^{22}$ noted that some ROMs can result in an inaccurate analysis. Madden et al. ${ }^{22}$ presented an algorithm to test and select a subset of the full IROM for obtaining an accurate result. The methods proposed herein allow for the MPS and damping methods to be implemented in the same algorithm by defining the modal matrix for each IROM.

\section{Conclusions}

Two methods used for a new damping identification were proposed. The optimized measurement point selection (MPS) method improves robustness of the identification to noise. Also, by taking advantage of cyclic symmetry, the computational cost of utilizing the MPS method is dramatically reduced. In addition, an algorithm was presented which describes an effective procedure incorporating the MPS method into experiment preparation.

The second method involves identifying the damping associated with each system mode. The benefits of the proposed method include the ability to maintain accuracy despite reducing the order of the models used to predict the system response and despite the fact that regions of high modal density are encountered. The proposed method was shown to be relatively insensitive to noise, to apply to mistuned systems, and to require knowledge of only the forced response, the relative forcing, and a finite element model.

The novel modal damping identification method was demonstrated using a numerical simulation of a low dimensional system and a more complex system (the University of Michigan validation blisk). Results involving several reduced order models (ROMs) of various sizes and various noise levels were presented. To increase the accuracy of the proposed method, the optimized MPS technique was implemented. Results indicate that using the optimal MPS technique increases the accuracy of the identification as compared to only using points chosen based on the (classical) EIDV method.

\section{Acknowledgment}

This work was supported by GE Aviation through the University Strategic Alliance program, with Steve Manwaring as the subsection manager and Dave Bulman as the section manager. The authors would also like to thank Sergio Filippi for insightful technical discussions.

\section{References}

${ }^{1}$ Penny, J. E. T., Friswell, M. I., and Garvey, S. D., "Automatic Choice of Measurement Locations for Dynamic Testing," AIAA Journal, Vol. 32, No. 2, 1994, pp. 407-414.

${ }^{2}$ Holland, D. E., Castanier, M. P., Ceccio, S. L., Epureanu, B. I., and Filippi, S., "Testing and Calibration Procedures for Mistuning Identification and Traveling Wave Excitation of Blisks," Journal of Engineering for Gas Turbines and Power, Vol. 132, No. 4, 2010, pp. 042502.

${ }^{3}$ Chen, S. Y., Ju, M. S., and Tsuei, Y. G., "Estimation of Mass, Stiffness and Damping Matrices from Frequency Response Functions," Journal of Vibration and Acoustics, Vol. 118, 1996, pp. 78-82.

${ }^{4}$ Adhikari, S. and Phani, A. S., "Experimental Identification of Generalized Propotional Viscous Damping Matrix," Journal of Vibration and Acoustics, Vol. 131, 2009, pp. 011008-1-011008-12. 
${ }^{5}$ Chen, S. Y., "A System Matrices Reduction Method for Damped Systems," JSME Internation Journal, Series C, Vol. 48, No. 4, 2005, pp. 681-687.

${ }^{6}$ Kim, K.-S., Kang, Y. J., and Yoo, J., "Structural Parameters Identification Using Improved Normal Frequency Response Function Method," Mechanical Systems and Siganl Processing, Vol. 22, 2008, pp. 1858-1868.

${ }^{7}$ Lancaster, P., "Expressions for Damping Matrices in Linear Vibration Problems," Journal of the Aerospace Sciences, Vol. 28, 1961, pp. 256.

${ }^{8}$ Adhikari, S., "Lancaster's Method for Damping Identification Revisited," Journal of Vibration and Acoustics, Vol. 124, 2002, pp. 617-627.

${ }^{9}$ Alvin, K. F., Peterson, L. D., and Park, K. C., "Extraction of Normal Modes and Full Modal Damping from Complex Modal Parameters," AIAA, Vol. 35, No. 7, 1997, pp. 1187-1193.

${ }^{10}$ Roemer, M. J. and Mook, D. J., "Mass, Stiffness, and Damping Matrix Identification: An Integrated Approach," Journal of Vibration and Acoustics, Vol. 114, 1992, pp. 358-363.

${ }^{11}$ Minas, C. and Inman, D. J., "Identification of a Nonproportional Damping Matrix from Incomplete Modal Information," Journal of Vibration and Acoustics, Vol. 113, 1991, pp. 219-224.

${ }^{12}$ Adhikari, S. and Woodhouse, J., "Identification of Damping: Part 1, Viscous Damping," Journal of Sound and Vibration, Vol. 243, No. 1, 2001, pp. 43-61.

${ }^{13}$ Adhikari, S. and Woodhouse, J., "Identification of Damping: Part 2, Non-viscous Damping," Journal of Sound and Vibration, Vol. 243, No. 1, 2001, pp. 63-88.

${ }^{14}$ Adhikari, S. and Woodhouse, J., "Identification of Damping: Part 3, Symmetry-Preserving Methods," Journal of Sound and Vibration, Vol. 251, No. 3, 2002, pp. 477-490.

${ }^{15}$ Adhikari, S. and Woodhouse, J., "Identification of Damping: Part 4, Error Analysis," Journal of Sound and Vibration, Vol. 251, No. 3, 2002, pp. 491-504.

${ }^{16}$ Lee, J. H. and Kim, J., "Development and Validation of a New Experimental Method to Identify Damping Matrices of a Dynamic System," Journal of Sound and Vibration, Vol. 246, No. 3, 2001, pp. 505-524.

${ }^{17}$ Yang, M.-T. and Griffin, J. H., "A Normalized Modal Eigenvalue Approach for Resolving Modal Interaction," Journal of Engineering for Gas Turbines and Power, Vol. 119, No. 3, 1997, pp. 647-650.

${ }^{18}$ Saito, A., Epureanu, B. I., Castanier, M. P., and Pierre, C., "Node Sampling for Nonlinear Vibration Analysis of Structures with Intermittent Contact," AIAA Journal, Vol. 48, No. 9, 2010, pp. 1903-1915.

${ }^{19}$ D'Souza, K., Saito, A., and Epureanu, B. I., "Reduced Order Modeling for Nonlinear Analysis of Cracked Mistuned Multi-Stage Bladed Disk Systems," AIAA Journal, (submitted for publication).

${ }^{20}$ Marinescu, O., Epureanu, B. I., and Banu, M., "Reduced-Order Models of Mistuned Cracked Bladed Disks," Journal of Vibration and Acoustics, (submitted for publication).

${ }^{21}$ D'Souza, K. and Epureanu, B. I., "A Statistical Characterization of the Effects of Mistuning in Multi-Stage Bladed Disks," Journal of Turbomachinery, (submitted for publication).

${ }^{22}$ Madden, A., Castanier, M., and Epureanu, B., "Reduced-Order Model Construction Procedure for Robust Mistuning Identification of Blisks," AIAA, Vol. 46, No. 11, 2009, pp. 2890-2898.

${ }^{23}$ Madden, A., Castanier, M. P., and Epureanu, B. I., "Mistuning Identification of Blisks at Higher Frequencies," Collection of Technical Papers AIAA/ASME/ASCE/AHS/ASC Structures, Structural Dynamics and Materials Conference, AIAA, 2010.

${ }^{24}$ Holland, D., Epureanu, B., and Filippi, S., "Damping Identification for Mistuned Blisks," Proceedings of the ASME International Design Engineering Technical Conferences, American Society of Mechanical Engineers, 2010, pp. 1-12.

${ }^{25}$ Judge, J., Pierre, C., and Ceccio, S., "Experimental Mistuning Identification in Bladed Disks using a Component-ModeBased Reduced-Order Model," AIAA Journal, Vol. 47, No. 5, 2009, pp. 1277-1287. 